\title{
Relationship of motility and acrosome reaction on sexing sperm in Ongole Crossbred bull
}

\author{
Aulia Puspita Anugra Yekti $^{1 *}$, Rifai Mustofa ${ }^{1}$ and Muhammad Lutfi $^{2}$ \\ ${ }^{1}$ Faculty of Animal Science, Universitas Brawijaya, Malang, Indonesia \\ ${ }^{2}$ Beef Cattle Research Station, Grati, Indonesia
}

\begin{abstract}
Artificial insemination using sexing semen is expected to produce calves with the desired sex. One sexing sperm method is the percoll density gradient centrifugation method. This study aimed to determine the changes and the relationship between motility and acrosome reaction after sexing process using percoll density gradient centrifugation. The material used was semen of \pm 5 years old Ongole crossbred bull with a bodyweight of $\pm 700 \mathrm{~kg}$ as many as three bulls with mass motility $2+$ and individual motility $70 \%$. The method used was to compare fresh semen with sexed semen after the cooling process. Parameters measured were motility characters using CASA analysis, which included motility parameters, progressive motility, capacitation, and no acrosome reaction. Statistical analysis used paired T-test to distinguish among fresh semen, after sexing and cooling process. In comparison, regression and correlation were used to analyze the relationship of capacitation and hyperactivation sperm with no acrosomal reaction with motility and progressive motility. The results showed that motility and progressive motility decreased after the sexing and cooling process. Meanwhile, the acrosomal reaction, capacitation, and hyperactivity increased.
\end{abstract}

\section{Introduction}

Artificial insemination using frozen semen sexing can increase the chances of producing male or female offspring [1, 2]. It was revealed by Susilawati et al. [3] that sexing spermatozoa using the Percoll density gradient centrifugation method resulted in a decrease in the quality of spermatozoa. It is because of damage to the spermatozoa membrane in the structure and function of the spermatozoa. Before the fertilization process, spermatozoa experience the biochemical and physiological structural modifications in the female reproductive organs, namely capacitation and acrosomal reactions [4].

Frozen semen has a lower fertility rate than fresh semen because the freezing process can damage the sperm plasma membrane and induce premature capacitation, increasing calcium ions [5]. The sexing process involves a centrifugation process that can reduce spermatozoa's quality to improve the capacitation of spermatozoa. Therefore, this research is conducted to see how the relationship between motility and capacitation and the acrosome reaction in semen after sexing.

\footnotetext{
*Corresponding author: auliapay@ub.ac.id
} 


\section{Materials and methods}

\subsection{Materials}

The semen used in this study was semen from 3 Ongole Crossbred bulls aged \pm 5 years with a bodyweight of $\pm 700 \mathrm{~kg}$. The semen met the minimum motility requirements of mass $2+$, individual motility $70 \%$.

\subsection{Methods}

The research method used was experimental method. Parameters measured were motility characters using CASA on parameters of motility, progressive motility, and hyperactivity. Observations of acrosome reactions included sperm that had not undergone acrosomal reaction (intact) and spermatozoa that had capacitation [6].

\subsection{Percoll density gradient centrifugation sexing}

The sexing method used was percoll density gradient centrifugation using 10 gradients of percoll. The diluent used was Andromed (Minitube Ref 13503/0200) with a ratio of 1:4 with aquabidest. Gradients were arranged in every $0.5 \mathrm{ml}$ from the bottom $65 \%$ to the top $20 \%$. Then $1 \mathrm{ml}$ semen was added into the tube gently. Then, it was centrifuged at 2250 rpm for 5 minutes. After centrifugation, $2 \mathrm{ml}$ of the bottom and $2 \mathrm{ml}$ of the top were taken and then put into different test tubes containing $3 \mathrm{ml}$ of wash andromed diluent. Furthermore, wash by centrifugation at $1500 \mathrm{rpm}$ for 3 minutes. Finally, remove the supernatant, and leave $1 \mathrm{ml}$ of precipitate in the tube. Then diluted to the total concentration of 25 million/straw and cooled at $5^{\circ} \mathrm{C}[7,8]$.

\subsection{Dilution and cooling of semen}

The sexed semen has then calculated the concentration using a hemocytometer and diluted to a concentration of 25 million/straw [9]. Then followed with the process of cooling to a temperature of $5^{\circ} \mathrm{C}$ in the refrigerator [7].

\subsection{Variable of research}

The variables observed in this study were:

1) Motility, progressive motility, and hyperactivation

Motility, progressive motility and hyperactivation were observed using CASA with Sperm Class Analyzer (SCA). Semen of 3-4 ul was placed on a warm object-glass with $37^{\circ} \mathrm{C}$ covered with a cover glass. The microscope was set at a magnification of $10 \times 10$ and phase contrast of $\mathrm{pH} 1$. The sperm was observed at 5 points of view. The results of the analysis will show the average value of the images obtained[10].

\section{Capacitation Assay}

The CTC stock solution containing $750 \mu \mathrm{M}$ CTC-HCl (Sigma), $130 \mathrm{mM} \mathrm{NaCl,} 5 \mathrm{mM}$ Lcysteine, $20 \mathrm{mM}$ Tris acid ( $\mathrm{pH} 7.8$ ) was prepared daily, wrapped in foil to protect against light, and stored at $4^{\circ} \mathrm{C}$ until required. $10 \mu \mathrm{L}$ of sperm were mixed with $15 \mu \mathrm{L}$ of CTC solution on a slide at room temperature. Then, $0.3 \mu \mathrm{L}$ of $12.5 \%$ glutaral $\neg$ dehyde in $2.5 \mathrm{M}$ 
Tris base was added as a fixative solution. Samples were covered with coverslips and stored in the dark room at $4^{\circ} \mathrm{C}$ [3]. The sperm were observed using an Olympus BX 53 fluorescent microscope. Non-capacitation spermatozoa will show fully bright fluorescence on the entire head. The capacitated spermatozoa will show fluorescence in the upper half of the head and dark in the lower half. In contrast, Spermatozoa with acrosomal reaction will show the minimal fluorescence signal with a bright signal in the equatorial region [11].

\subsection{Data analysis}

The statistical analysis used paired T-test to distinguish between fresh semen after sexing and cooling process. In comparison, regression and correlation were used to analyse the relationship of capacitation and hyperactivation sperm with no acrosomal reaction with motility and progressive motility.

\section{Results and discussion}

\subsection{Motility, capacitation, no acrosomal reaction, and hyperactivity in fresh semen and after sexing}

The quality of sperm in the fresh semen and after sexing, including motility, capacitation, no acrosomal reaction, and hyperactivation are presented in table 1.

Table 1. Characteristics of motility and acrosome reactions in fresh semen and after sexing and cooling

\begin{tabular}{|c|c|c|}
\hline Parameter & Fresh semen & Sexing semen \\
\hline Motility (\%) & $95,05 \pm 5,16 \%$ & $71,02 \pm 10,08^{* *}$ \\
\hline Progresif Motility (\%) & $71,13 \pm 5,82 \%$ & $47,68 \pm 8,71^{* *}$ \\
\hline Hiperactivation (\%) & $11,30 \pm 4,83 \%$ & $12,96 \pm 6,35 \%^{\mathrm{ns}}$ \\
\hline Capacitation & $21,51 \pm 11,47 \%$ & $24,90 \pm 9,38^{\mathrm{ns}}$ \\
\hline no acrosomal reaction (\%) & $74,28 \pm 13,15 \%$ & $69,60 \pm 9,59^{*}$ \\
\hline
\end{tabular}

Note : $* *$ In the same row showed the very significant differences $(\mathrm{P}<0.01)$

* In the same row showed the significant differences $(\mathrm{P}<0.05)$

The percentage of motility of fresh semen was $95.05 \pm 5.16 \%$, while after sexing $71.02 \pm$ $10.08 \%$, the percentage of motility experienced a very significant decrease $(\mathrm{P}<0.01)$ after the sexing and cooling process. The percentage of progressive motility in fresh semen was $71.13 \pm 5.82 \%$, after sexing and cooling was decreased significantly $(\mathrm{P}<0.01)$ to $47.68 \pm$ $8.71 \%$. This is because the sexing process, which involves centrifugation, causes damage to the sperm membrane, resulting in decreased motility after sexing [3] [7]. The cooling process also results in changes in the protein structure of the sperm. Cold temperatures cause a restriction of the lateral movement of phospholipids from the bilayer membrane, which induces a change in shape from the liquid to the gel phase, which causes the membrane to become more rigid and brittle. This causes the separation of the lipid phase resulting in irreversible protein buildup [12]. Protein has a crucial role in the life of sperm, the fertilization process, and energy metabolism [13].

Spermatozoa capacitation in fresh condition was $21.51 \pm 11.47 \%$, while after sexing and cooling to $24.90+9.38$, the capacitation value increased not significantly $(\mathrm{P}>0.05)$. The picture of capacitation using CTC staining showed an increase in calcium ions at the equator and the neck of the spermatozoa. 
Hyperactivation of spermatozoa in fresh semen was $11.30 \pm 4.83 \%$, whereas after sexing and cooling, it became $12.96 \pm 6.35 \%$, the hyperactivated spermatozoa had no significant increase $(\mathrm{P}>0.05)$. The centrifugation process in sexing and the chemicals present in the diluent induce the membrane to influx calcium ions and capacitation occurs. The cooling and freezing process causes a decrease in membrane integrity, increased capacitation and calcium ion levels [5]. Increased calcium leads to increased hyperactivation by activating the signal transduction cascade regulating hyperactivation. Calcium ions will interact with the axoneme of flagellum to trigger hyperactivation [14].

From the study results, spermatozoa that sperm with no acrosomal reaction in fresh semen were $74.28 \pm 13.15 \%$ and after cooling and sexing were $69.60 \pm 9.59 \%$. This indicates that during the sexing and cooling process, there was an increase in the acrosome reaction significantly $(\mathrm{P}<0.05)$. In mammals, capacitation is followed by a direct acrosomal reaction, and then the spermatozoa are ready to fertilize the egg [15].

\subsection{Relationship between capacitation and hyperactivity}

In fresh condition, the capacitation value was $21.51 \pm 11.47 \%$, while hyperactivity was $11.30 \pm 4.83 \%$, the relationship between capacitation and hyperactivity was $\mathrm{Y}=-0.0134 \mathrm{X}$ $+12,179$ with $r=0.0008$. This equation shows a positive relationship between capacitation and hyperactivity. This shows that the capacitation value is inversely proportional to the hyperactivity value. When the capacitation value increases, the hyperactivity value will increased. After being capacitated, spermatozoa can bind to the zona pellucida through the acrosome reaction (AR), followed by penetration and fertilization of the oocyte. Capacitation is characterized by actin polymerization and will develop hyperactive motility (HAM) [16]. However, the value of $r=0.0008$ indicates that the correlation between the capacitation value and the hyperactivity value in fresh spermatozoa is weak because normally semen will undergo capacitation on the female reproductive tract before fertilization process[4].

After sexing and cooling process, the capacitation value was $25.8 \pm 8.6 \%$, while hyperactivity was $12.96 \pm 6,35 \%$, the relationship between capacitation and hyperactivity was $y=0.1297 x+9.604, r=0.0311$. The same as fresh condition, the correlation equation between capacitation and hyperactivity in conditions after sexing and cooling showed a positive value. That is, an increase in in capacitation will be followed by an increase in hyperactivity. However, the value of $r=0.0311$ still shows that the correlation between capacitation and hyperactivity value is very weak. This is not different from the correlation between capacitation value and hyperactivity value in fresh semen. The cooling process of semen causes sperm damage, including ion imbalance, protease activation, cellular acidosis, energy deficiency, membrane phase transition, and the production of free radicals or reactive oxygen species (ROS) [12]. ROS are free radicals that affect the physiology of spermatozoa during capacitation and hyperactivation [17]. Capacitation of sperm is followed by the increase of ROS and leads to a redox signaling cascade. Redox modifications of proteins accompany bull sperm capacitation [11].

\subsection{The relationship between the acrosome reaction with motility and progressive motility}

In the fresh semen of spermatozoa that have not reacted acrosome $74.28 \pm 13.15 \%$ with motility $95.05 \pm 5.16 \%$, has a regression relationship $y=0.0499 x+91.35, r=0.0162$. The regression equation shows a correlation between acrosome reaction and motility. It is 
interesting that with the acrosome reaction, the motility of spermatozoa is higher. Otherwise, a decreased value of the acrosome reaction will be followed by a decrease motility. However, the correlation equation showed a very weak relationship between the acrosome reaction and the motility value of the fresh spermatozoa. This is indicated by the value of $r=0.0162$.

Fresh semen also showed a similar correlation between spermatozoa with no acrosomal reaction with progressive motility of $71.13 \pm 5.82 \%$. The regression equation shows $y=0,1069+63,186$ with $r=0,05833$. The positive value in the regression equation showed that an increase of acrosome reaction would follow an increase in progressive motility. However, the value of $r=0.05833$ indicates a very weak relationship between the acrosome reaction and the progressive motility of fresh spermatozoa in this study.

In sexing sperm, spermatozoa that have no acrosome reaction $69.60 \pm 9.59 \%$, with motility $71.02 \pm 10.08 \%$, have a regression relationship $Y=-0.7322 \mathrm{x}+121.98, \mathrm{r}=0.48456$. This equation shows a negative value that indicates an opposite relationship between the value of spermatozoa without acrosome reaction and the motility of sexing spermatozoa after cooling. This indication shows that with the increasing value of the acrosome reaction, the value of motility will decrease, ultimately reducing the ability to fertilize [5]. The equation also shows a strong relationship between changes in the acrosome reaction value and the motility value of sexing semen after cooling. The value of $r=0.48456$ indicates a fairly strong relationship between changes in the value of the acrosome reaction and the value of motility.

A negative regression relationship was also shown in the semen value after sexing and cooling between spermatozoa that had no acrosomal reaction with progressive motility $47.68 \pm 8.71 \%, \mathrm{Y}=-0.1295 \mathrm{x}+56.69, \mathrm{r}=0.02033$. Thus the value of spermatozoa with no acrosomal reaction is inversely proportional to the progressive motility in conditions after sexing and cooling. The value of progressive motility will decrease if the acrosome reaction increases, and conversely, the value of progressive motility will increase if the acrosomal reaction decreases. However, in contrast to the relationship between acrosomal reaction values and motility values, which showed a strong relationship, the regression equation for acrosomal reaction values and progressive motility did not show a strong relationship. This is indicated by the value of $r=0.02033$.

\section{Conclusion}

Motility and progressive motility decreased after the sexing and cooling process. The process of sexing and cooling sperm causes increased capacitation, acrosomal reactions, and hyperactivation.

\section{References}

1. M.A. Prakash, Advances in Animal and Veterinary Sciences, 2, 226-232 (2014).

2. P. Boro et al., International Journal of Applied Research, 2, 460-462 (2016).

3. T. Susilawati et al., Advances in Health Sciences Research (AHSR), 1st International Conference in One Health (ICOH), 5, 1-5 (2017).

4. D. Ickowicz, M. Finkelstein, and H. Breitbart, Asian J Androl, 14, 816-821 (2012).

5. S. Collin et al., Journal of Andrology, 21, 938-943 (2000).

6. R. Mustofa et al., Jurnal Agripet, 20, 210-215 (2020).

7. E.D. Kusumawati et al., American Journal of Animal and Veterinary Sciences, 14, 111-114 (2019). 
8. T. Susilawati, Sexing Spermatozoa (2014).

9. T. Susilawati, Pedoman Inseminasi Buatan Pada ternak, (2013).

10. D. Ratnawati, N. Isnaini, and T. Susilawati, Jurnal Ilmu-Ilmu peternakan, 27, 80-95 (2017).

11. A. Mostek et al., Int J Mol Sci, 22, 1-21 (2021).

12. J.G. Baust, JD. Gao, and J.M. Baust, Organogenesis, 5, 90-6 (2009).

13. A.A. Moura and E. Memili, Animal Reproduction, 13, 191-199 (2016).

14. S.S. Suarez and H.C. Ho, Reprod Domest Anim, 38, 119-124 (2003).

15. N. Hirohashi and R. Yanagimachi, Biol Reprod, 99, 127-133 (2018).

16. S.B. Itach et al., Dev Biol, 362, 154-161 (2012).

17. R.J. Aitken, K.T. Jones, and S.A. Robertson, J Androl, 33, 1096-106 (2012). 